\title{
The influence of post-fixation on visualising vimentin in the retina using immunofluorescence method
}

\author{
B. Baykal ${ }^{1}$, C. Korkmaz ${ }^{1}$, N. Kocabiyik² ${ }^{2}$ O.M. Ceylan ${ }^{3}$ \\ ${ }^{1}$ Department of Histology and Embryology, Gulhane Faculty of Medicine, University of Health Sciences, Ankara, Turkey \\ 2Department of Anatomy, Gulhane Faculty of Medicine, University of Health Sciences, Ankara, Turkey \\ ${ }^{3}$ Department of Ophthalmology, Diskapi Yildirim Beyazit Training and Research Hospital, \\ University of Health Sciences, Ankara, Turkey
}

[Received: 9 June 2017; Accepted: 24 July 2017]

Background: Post-fixation of sections is especially required for cryostat sections of fresh frozen tissues. Vimentin is an intermediate filament in both fibrillary and non-fibrillary form, expressed in Müller's cells and astrocytes of the retina. Our aim was to determine the best post-fixation method for visualising vimentin in archival mouse eyes.

Materials and methods: We used an archival mouse eye, slightly pre-fixed with paraformaldehyde and stored at $-80^{\circ} \mathrm{C}$ for 4 years. We tried three fixatives (paraformaldehyde [PFA], alcohol/acetic acid [AAA] and methanol) for post-fixation of eye sections.

Results: We showed that post-fixation alters the labelling properties of vimentin expressed in the retina. In the sections with no post-fixation, vimentin positivity was observed in and around the nuclei in non-fibrillary form. In PFA post-fixed sections, the vimentin in the retina was not observed as fibrils. Positivity was observed in the nuclei and in perinuclear regions of the cells. In AAA post-fixed sections, positive labelling was observed around the nuclei as fibrils. In methanol post-fixed sections, labelling was observed around the nuclei as fibrils.

Conclusions: We conclude that post-fixation with AAA is more convenient for immunofluorescent labelling of vimentin in the retina for slightly PFA pre-fixed and long-term stored retina. (Folia Morphol 2018; 77, 2: 246-252)

Key words: vimentin, post-fixation, paraformaldehyde, alcohol/acetic acid, methanol

\section{INTRODUCTION}

Vimentin is a $57 \mathrm{kDa}$ intermediate filament expressed in cells of mesenchymal origin [5]. It is located both in the cell cytoplasm and nucleus [10]. It can be present in fibrillary and non-fibrillary forms [11]. Vimentin is the major cell skeleton component in the immature glial cells of the rat brain [4]. In the adults, it is expressed in Müller's cells and astrocytes of the retina $[3,16,19]$.

The eyeball consists of three layers: corneoscleral layer (includes sclera and cornea), vascular layer (uvea) and retina. Retina is arranged into ten layers. These layers are retinal pigment epithelium (RPE), rod and cone cells layer (RCL), outer limiting membrane (OLM),

Address for correspondence: Prof. Dr. B. Baykal, Department of Histology and Embryology, University of Health Sciences, Ankara, Turkey, e-mail: baris_baykal@yahoo.com 
outer nuclear layer (ONL), outer plexiform layer (OPL), inner nuclear layer (INL), inner plexiform layer (IPL), ganglion cell layer (GCL), nerve fibre layer (NFL) and inner limiting membrane (ILM). The cell bodies of Müller's cells are localised in the INL and their processes extend to OLM and ILM. Thus, the processes of Müller's cells traverse almost the entire retina [17]. Astrocytes are localised in NFL and GCL [16].

The expression of vimentin increases after a mechanical damage like retinal detachment [15]. In addition, the vimentin positivity in the processes of Müller's cells, which extend through the both borders of retina, reaches the deep layers of retina after retinal detachment [14]. Together with the vimentin expression in cell bodies and processes of the astrocytes, vimentin expression in the retina extends from the NFL to ONL under pathologic conditions [16].

Post-fixation is a method, which is performed by immersion of sections of fresh frozen tissues into a fixative especially after being sectioned by a cryostat. An increase in staining intensity has been reported after this method [18]. Perfusion fixation with glutaraldehyde and post-fixation with osmium tetroxide is a method used for electron microscopy [21].

During a study on vimentin expression in slightly paraformaldehyde (PFA) pre-fixed and long-term cryopreserved archival retina, we encountered difficulty in visualisation of vimentin positivity. Thinking that it may be due to the fixative used for post-fixation, we planned this study in which we tried post-fixation with three fixatives.

\section{MATERIALS AND METHODS}

\section{The source of archival tissues}

The eye used in this study is an archival tissue from a male Swiss-Webster mice (100 days old), which undergone acute carbon monoxide (CO) poisoning in a previous study, which was approved by the local ethical committee (July 06, 2005; approval number: 05/68; Animal Research Ethics Committee of Gulhane Military Medical Faculty). Also, this study on archival tissues was approved by the local ethical committee (January 21, 2011; approval number: 11/1; Animal Research Ethics Committee of Gulhane Military Medical Faculty). Acute carbon monoxide poisoning was performed using the method described by Gilmer et al. [7]. Briefly, the animal first inhaled 1000 ppm. CO for $40 \mathrm{~min}$ and then 50,000 ppm. CO until the loss of consciousness. This animal was perfusion fixed by first infusion of physiological saline solution and then a $4 \%$ solution of PFA in phosphate buffered saline (PBS) under general anaesthesia with xylazine/ketamine. After an in-situ waiting time of $30 \mathrm{~min}$, its brain was used and the eyes were preserved at $-80^{\circ} \mathrm{C}$ for 4 years after an overnight incubation in $30 \%$ sucrose solution in PBS at $4^{\circ} \mathrm{C}$.

\section{Sectioning}

The eye was first kept inside the cryostat (Shandon, Thermo Electron Corporation) at $-25^{\circ} \mathrm{C}$ for $30 \mathrm{~min}$ for thermal equilibration and then serial sections at a thickness of $25 \mu \mathrm{m}$ were taken on 4 slides in a systematically random manner. Each of the 4 slides included 8-10 sections of the same eye. Thus, at least 5-6 sections were analysed for each of the post-fixation method.

\section{Post-fixation}

Three different post-fixation fixatives were applied to the sections on three slides. For control purposes postfixation was not applied to fourth slide. PFA was used on one of the slides, the other one received alcohol/acetic acid (AAA) (2/1, v/v) and the other one received methanol for post-fixation. Post-fixation with PFA was performed at room temperature for $5 \mathrm{~min}$. Post-fixation with AAA and methanol was performed at $-20^{\circ} \mathrm{C}$ for $5 \mathrm{~min}$.

\section{Labelling}

The sections were washed with PBS for $3 \times 5 \mathrm{~min}$ and were incubated in blocking solution ( $5 \%$ bovine serum albumin and 1\% Triton X-100 in PBS) for $2 \mathrm{~h}$. After that, the sections were incubated overnight (17 h) in blocking solution containing Cy3 conjugated anti-vimentin antibody (Sigma-Aldrich, Cat\# C9080) at a dilution of $1 / 20$ at $4^{\circ} \mathrm{C}$. After washing with PBS for $3 \times 5$ min, counterstaining was performed by incubating in PBS containing Hoechst 33258 at a dilution of $1 / 500$ for $5 \mathrm{~min}$. The sections were coverslipped with PBS containing $50 \%$ glycerine and sealed with nail polish.

\section{Imaging}

Imaging was performed under a fluorescent microscope (DMI6000B, Leica, Germany) which was equipped with appropriate filter cubes for $\mathrm{Cy} 3$ and Hoechst 33258. Densitometric analysis was performed using the same imaging software (Leica Application Suite Advanced Fluorescence, Leica, Germany).

\section{RESULTS}

All eye sections used in this study were from the same eye and at least 6 sections, which included 


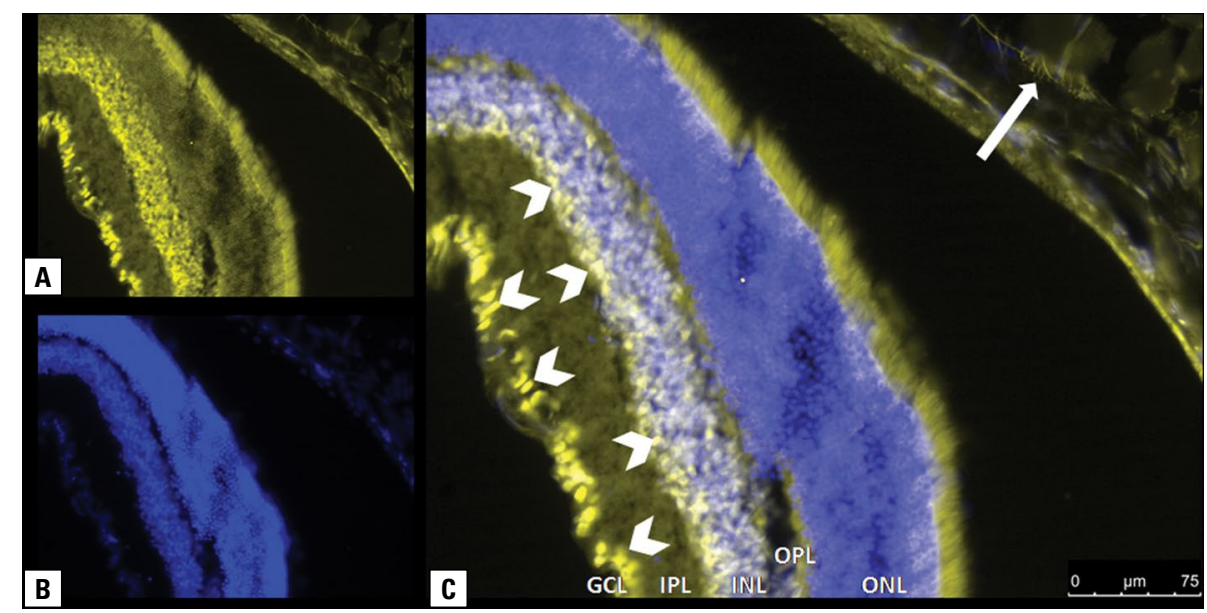

Figure 1. A section with no post-fixation. A. Vimentin labelled with Cy3; B. Nuclei stained with Hoechst; C. Overlay of panels A and B. Nuclear and perinuclear positive labelling is observed in ganglion cell layer (GCL) and inner nuclear layer (INL) of the retina (arrow heads). Positive labelling in fibrillary form is observed in the sclera (arrow). IPL — inner plexiform layer; ONL — outer nuclear layer; OPL — outer plexiform layer.

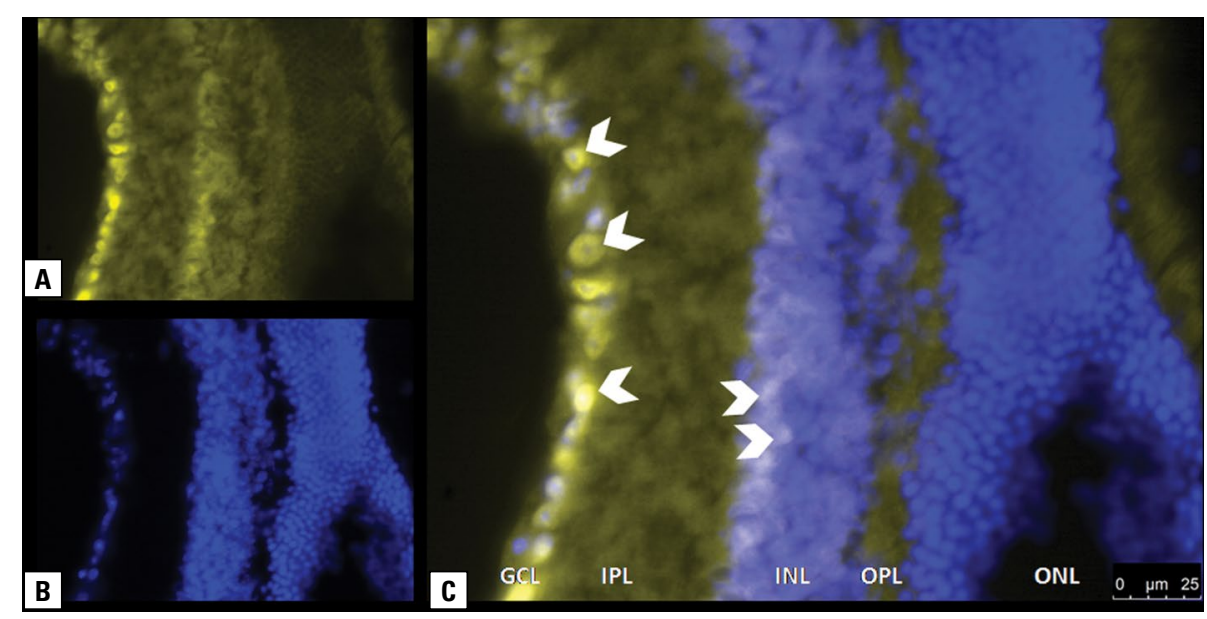

Figure 2. Retina in a paraformaldehyde post-fixed section. A. Vimentin labelled with Cy3; B. Nuclei stained with Hoechst; C. Overlay of a panels $A$ and $B$. Nuclear and perinuclear positive labelling is observed in ganglion cell layer (GCL) and inner nuclear layer (INL) of the retina (arrow heads); IPL — inner plexiform layer; ONL — outer nuclear layer; OPL — outer plexiform layer.

sections of retina, were analysed for each one of post-fixation method.

1. In the sections that were not post-fixed, vimentin positivity was observed in and around the nuclei of GCL and INL of the retina. No fibrillary organisation was observed inside the retina. The vimentin in the sclera and the connective tissue surrounding the eyeball was observed as fibrils (Fig. 1).

2. In the sections post-fixed with PFA, the vimentin in the retina was not observed as fibrils. Positivity was observed in the nuclei and in perinuclear regions of the cells of GCL and INL (Fig. 2). In the surrounding connective tissue vimentin was observed as fibrils. Also positive regions were observed in striated muscles (Fig. 3).
3. In the sections post-fixed with AAA, positive labelling was observed around the nuclei of GCL and vimentin was observed as fibrils extending from NFL to INL (Fig. 4). In the surrounding connective tissue vimentin was also observed as fibrils (Fig. 5).

4. In the sections post-fixed with methanol, labelling was observed around the nuclei and vimentin was observed as fibrils extending to INL. In addition to that, intensive positivity was observed in INL and OPL (Fig. 6). Vimentin is observed as fibrils in the sclera (Fig. 7).

5. According to densitometric analysis of images of AAA and methanol post-fixed sections, the peaks are more regular and the background noise is lower in AAA post-fixed sections (Fig. 8). 


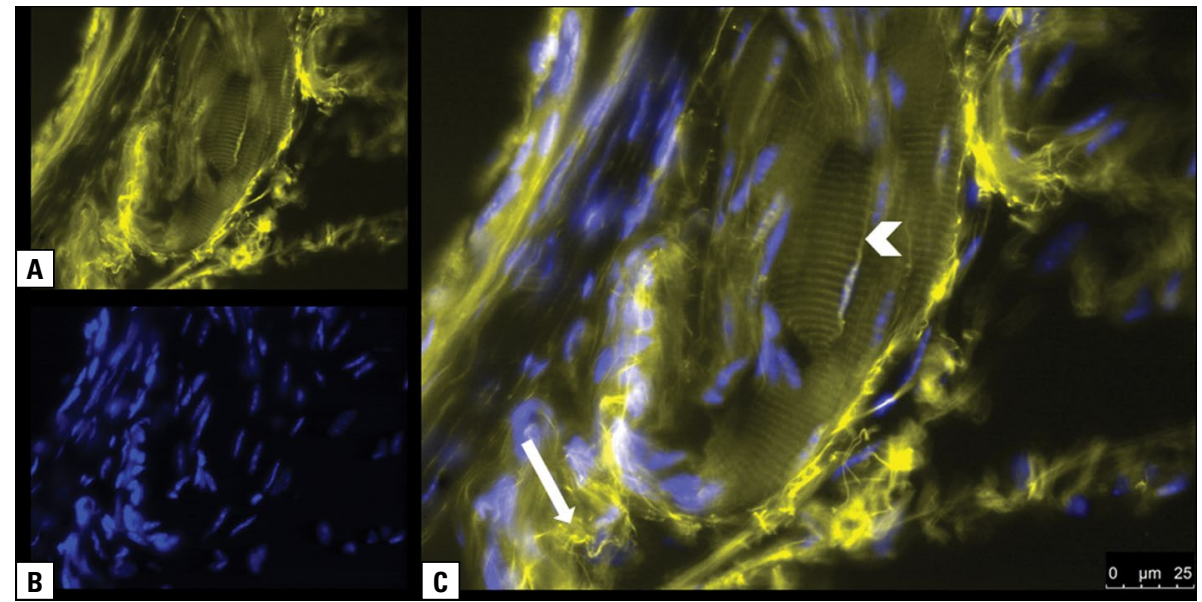

Figure 3. Connective and muscle tissues in a section post-fixed with paraformaldehyde. A. Vimentin labelled with Cy3; B. Nuclei stained with Hoechst; C. Overlay of a panels A and B. Fibrils in the connective tissue (arrow) and non-fibrillary vimentin in the striated muscle tissue can be observed (arrow head).

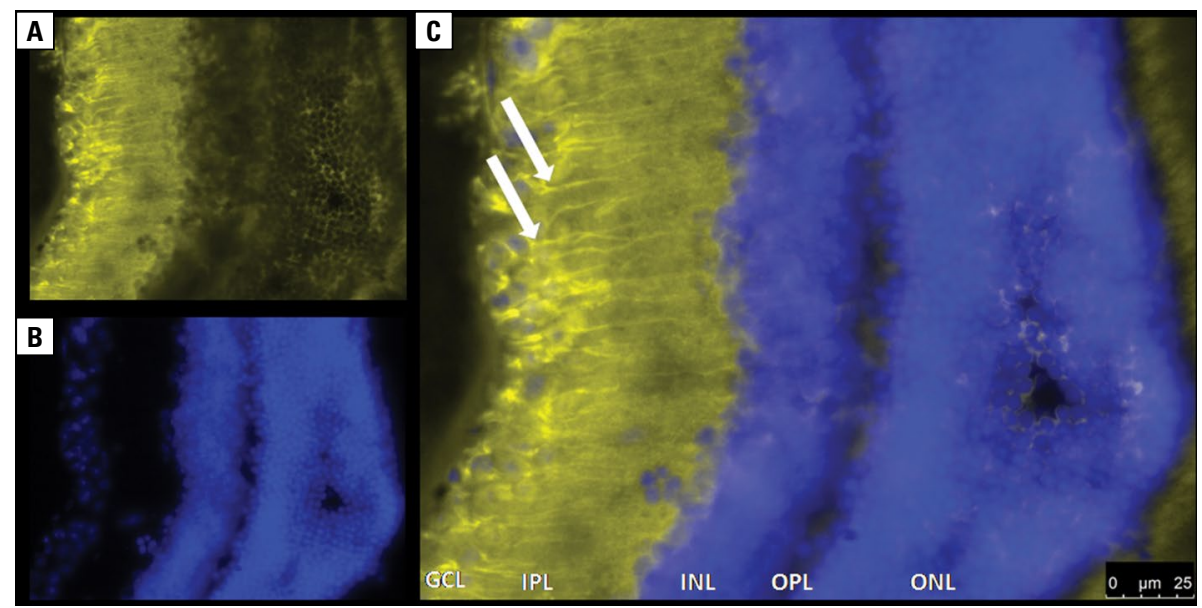

Figure 4. Retina in a section post-fixed with alcohol/acetic acid. A. Vimentin labelled with Cy3; B. Nuclei stained with Hoechst; C. Overlay of a panels $A$ and $B$. Vimentin is extending from ganglion cell layer (GCL) to inner nuclear layer (INL) as fibrils (arrows). IPL — inner plexiform layer; ONL — outer nuclear layer; OPL — outer plexiform layer.

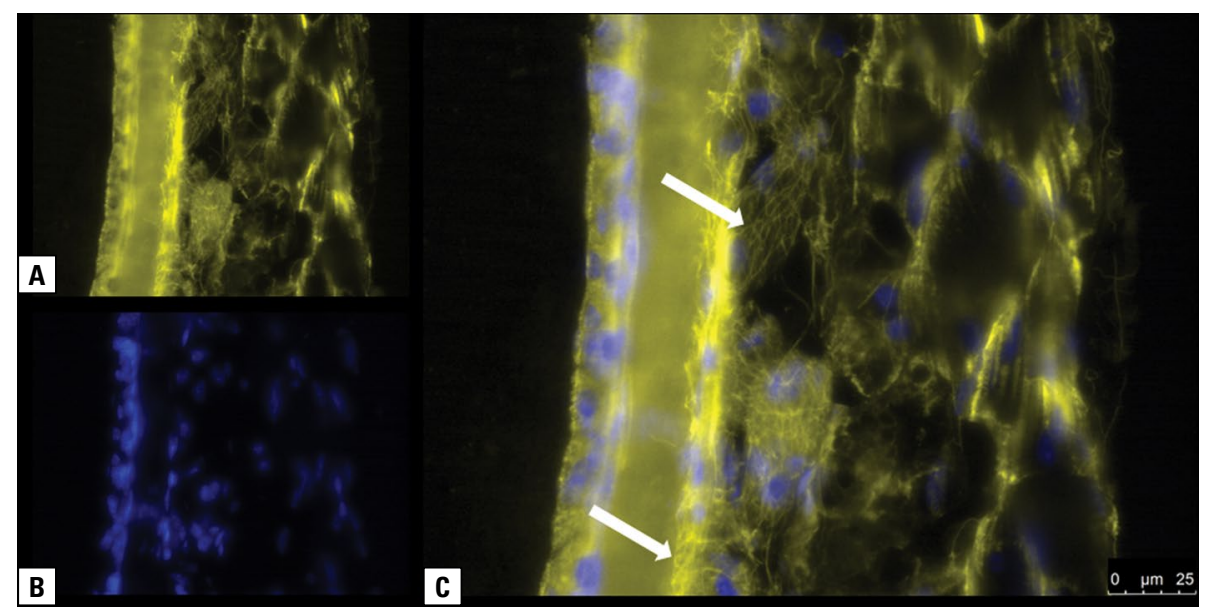

Figure 5. Sclera and connective tissue in a section post-fixed with alcohol/acetic acid. A. Vimentin labelled with Cy3; B. Nuclei stained with Hoechst; C. Overlay of panels A and B. Vimentin positive labelling is observed in the form of fibrils (arrows). 


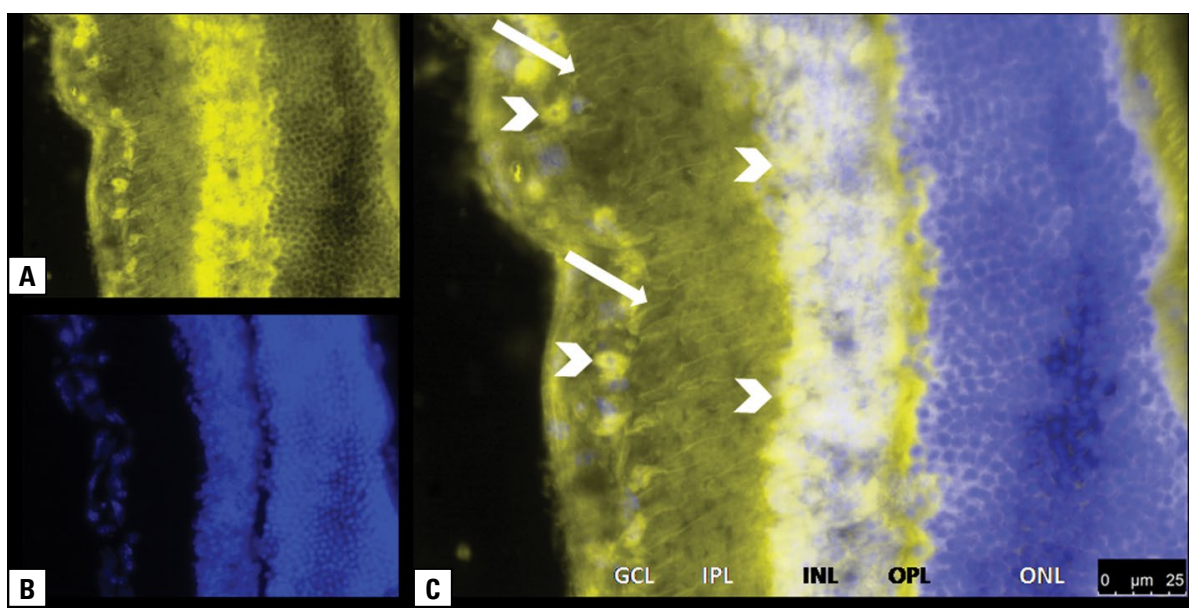

Figure 6. Retina in a section post-fixed with methanol. A. Vimentin labelled with Cy3; B. Nuclei stained with Hoechst; C. Overlay of panels $A$ and B. Nuclear and perinuclear positive labelling (arrow heads) is observed in ganglion cell layer (GCL) and inner nuclear layer (INL). Positive labelling in fibrillary form extending from GCL to INL (arrows) is also observed. Increased intensity is observed in outer plexiform layer (OPL); $\mathrm{IPL}$ — inner plexiform layer; ONL — outer nuclear layer.

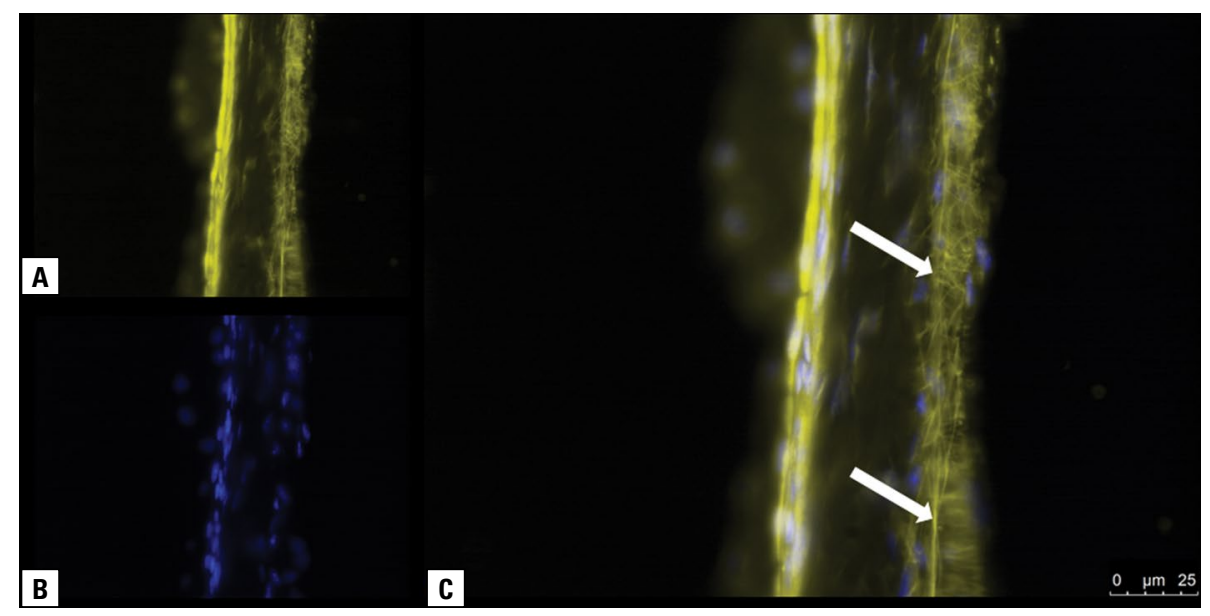

Figure 7. Sclera in a section post-fixed with methanol. A. Vimentin labelled with Cy3; B. Nuclei stained with Hoechst; C. Overlay of panels A and B. Positive labelling in fibrillary form is observed (arrows).

\section{DISCUSSION}

In this study, immunofluorescent vimentin labelling is performed on sections taken from an archival eye, which was slightly fixed via perfusion-fixation with PFA and preserved at $-80^{\circ} \mathrm{C}$ for 4 years. Although no statement regarding interaction with mouse tissues takes place in the user manual of the antibody used, the producer has stated a cross-reaction with mouse vimentin had been detected in the first lot of product. During trial labellings, because post-fixation was performed with PFA, fibrillary labelling has not been observed in the retina. However, positive labelling in the surrounding tissue and positive labelling in the retina after appropriate post-fixation has proved that the antibody works on mouse tissues. We used sections from a single eye and therefore we kept only the post-fixation step as a variable and all other conditions constant.

Fresh frozen sections tend to break. In order to prevent this, incubation in sucrose is required for taking good sections and good images. But, incubation in sucrose can only be performed if the tissue is pre-fixed, because, for sucrose to infiltrate the tissue, an overnight incubation is required and this time period causes unfixed tissue to be degraded by its own lysosomal enzymes. The 30 min pre-fixation 


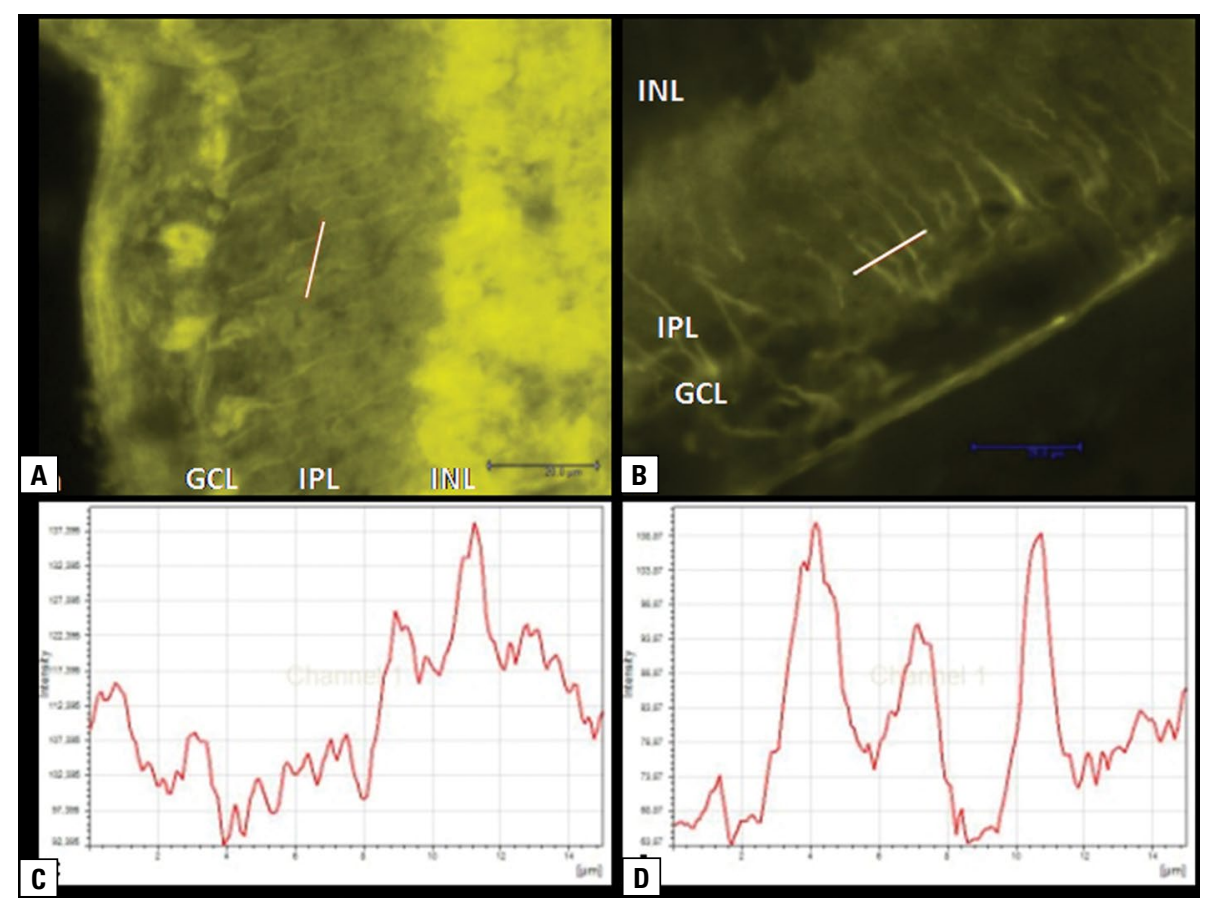

Figure 8. Densitometric analysis of fibrils positively labelled after methanol (A, C) and alcohol/acetic acid (AAA) (B, D) post-fixation. Intensity values of pixels on the lines are shown as histograms (C, D). The peaks in section post-fixed with AAA (D) are more regular and background noise is observed to be lower. GCL — ganglion cell layer; INL — inner nuclear layer; IPL — inner plexiform layer; ONL — outer nuclear layer; $\mathrm{OPL}$ - outer plexiform layer.

is a slight fixation because, for a good fixation with PFA, the tissues are usually offered to be immersed in it at least overnight.

Because fixation changes the chemical properties of tissue components and the three-dimensional conformation of proteins, it has important effects on the affinity and selectivity of the antibodies [6]. For this reason, optimum conditions must be detected for every molecule of interest. Alves et al. (1992) [1] have stated that different fixatives may be required for different epitops and has reported better interaction for vimentin in sections fixed with ethanol than those fixed with formalin. Helfand et al. (2011) [11] have suggested that methanol fixation is optimal for visualisation of vimentin. However, methanol is reported to cause protein coagulation, which results in decreased antigenicity and loss of cell architecture [12]. Boon and Kok [2] have reported that post-fixation with formalin causes false-negative immune labelling for vimentin. An interesting finding in our study is that, positive labelling for vimentin has been observed after postfixation with all three fixatives and also without postfixation, but variations have been observed in vimentin labelling in the retina according to the post-fixation method. We observed positive regions for vimentin in the striated muscles, which was also reported by Granger and Lazarides [8].

Fixation with PFA before labelling vimentin in the retina has been used in several studies $[13,14,20]$. In these studies the specimens have directly undergone labelling processes. Guidry et al. (2002) [9], on a study on human retina, have used PFA pre-fixation for $6 \mathrm{~h}$ and after sectioning the retina, they have stored the slides at $-20^{\circ} \mathrm{C}$. Before immunofluorescence experiments, they have warmed the slides to room temperature and post-fixed them with acetone for $3 \mathrm{~min}$. According to our findings, it is obvious that the prefixation and storing at $-80^{\circ} \mathrm{C}$, has provided enough preservation for vimentin and it is also obvious that the fixative used for post-fixation has an effect on visualisation of vimentin as fibrils. Researchers who have difficulty in visualisation of vimentin should try pre- and post-fixation methods mentioned here and in other studies.

\section{CONCLUSIONS}

In this study we tried three fixatives for postfixation of archival slightly PFA pre-fixed eye sections 
for labelling vimentin in the retina. In sections which were not post-fixed and those post-fixed with PFA, vimentin was not visible as fibrils. In the sections post-fixed with AAA or methanol, vimentin was visible as fibrils. Densitometric analysis of the images from AAA and methanol post-fixed sections revealed that AAA post-fixation yields more regular peaks and less background noise. In conclusion, for studies in which vimentin expression in the retina has to be shown extending to the outer layers of the retina in a fibrillary manner, AAA post-fixation is more convenient. For antibodies which are thought not to work, trials must be performed with combinations of variables in every step. It should also be noted that vimentin could be visualised using immunofluorescence even after storing of tissues perfusion-fixed with PFA at $-80^{\circ} \mathrm{C}$ for 4 years.

\section{Acknowledgements}

This study has been supported by The Scientific and Technological Research Council of Turkey (TUBITAK) (Grant No: 110S308).

\section{REFERENCES}

1. Alves VA, Wakamatsu $A$, Kanamura CT, et al. [The importance of fixation in immunohistochemistry: distribution of vimentin and cytokeratins in samples fixed in alcohol and formol]. Rev Hosp Clin Fac Med Sao Paulo. 1992; 47(1): 19-24, indexed in Pubmed: 1284893.

2. Boon ME, Kok LP. Formalin is deleterious to cytoskeleton proteins: do we need to replace it by formalin-free Kryofix? Eur J Morphol. 1991; 29(3): 173-180, indexed in Pubmed: 1726665.

3. Dahl D, Bignami A, Weber K, et al. Filament proteins in rat optic nerves undergoing Wallerian degeneration: localization of vimentin, the fibroblastic 100-A filament protein, in normal and reactive astrocytes. Exp Neurol. 1981; 73(2): 496-506, indexed in Pubmed: 7021171.

4. Dahl D, Rueger DC, Bignami A, et al. Vimentin, the 57000 molecular weight protein of fibroblast filaments, is the major cytoskeletal component in immature glia. Eur J Cell Biol. 1981; 24(2): 191-196, indexed in Pubmed: 7285936.

5. Franke WW, Schmid E, Osborn M, et al. Different intermediate-sized filaments distinguished by immunofluorescence microscopy. Proc Natl Acad Sci U S A. 1978; 75(10): 5034-5038, doi: 10.1073/pnas.75.10.5034.

6. Fritschy JM. Is my antibody-staining specific? How to deal with pitfalls of immunohistochemistry. Eur J Neurosci. 2008; 28(12): 2365-2370, doi: 10.1111/j.14609568.2008.06552.x, indexed in Pubmed: 19087167.

7. Gilmer B, Kilkenny J, Tomaszewski C, et al. Hyperbaric oxygen does not prevent neurologic sequelae after carbon monoxide poisoning. Acad Emerg Med. 2002; 9(1): 1-8, indexed in Pubmed: 11772662.
8. Granger BL, Lazarides E. Desmin and vimentin coexist at the periphery of the myofibril Z disc. Cell. 1979; 18(4): 1053-1063, doi: 10.1016/0092-8674(79)90218-6, indexed in Pubmed: 391403.

9. Guidry C, Medeiros NE, Curcio CA. Phenotypic variation of retinal pigment epithelium in age-related macular degeneration. Invest Ophthalmol Vis Sci. 2002; 43(1): 267-273, indexed in Pubmed: 11773041.

10. Hartig R, Shoeman RL, Janetzko A, et al. DNA-mediated transport of the intermediate filament protein vimentin into the nucleus of cultured cells. J Cell Sci. 1998; 111 (Pt 24): 3573-3584, indexed in Pubmed: 9819349.

11. Helfand BT, Mendez MG, Murthy SN, et al. Vimentin organization modulates the formation of lamellipodia. Mol Biol Cell. 2011; 22(8): 1274-1289, doi: 10.1091/mbc.E10-08-0699, indexed in Pubmed: 21346197.

12. Hoetelmans RW, Prins FA, Cornelese-ten Velde I, et al. Effects of acetone, methanol, or paraformaldehyde on cellular structure, visualized by reflection contrast microscopy and transmission and scanning electron microscopy. Appl Immunohistochem Mol Morphol. 2001; 9(4): 346-351, indexed in Pubmed: 11759062.

13. Johansson UE, Eftekhari S, Warfvinge K. A battery of celland structure-specific markers for the adult porcine retina. J Histochem Cytochem. 2010; 58(4): 377-389, doi: 10.1369/ jhc.2009.954933, indexed in Pubmed: 20086234.

14. Lewis GP, Fisher SK. Müller cell outgrowth after retinal detachment: association with cone photoreceptors. Invest Ophthalmol Vis Sci. 2000; 41(6): 1542-1545, indexed in Pubmed: 10798674.

15. Nakazawa T, Takeda M, Lewis GP, et al. Attenuated glial reactions and photoreceptor degeneration after retinal detachment in mice deficient in glial fibrillary acidic protein and vimentin. Invest Ophthalmol Vis Sci. 2007; 48(6): 2760-2768, doi: 10.1167/iovs.06-1398, indexed in Pubmed: 17525210.

16. Rehak $M$, Hollborn $M$, landiev I, et al. Retinal gene expression and Müller cell responses after branch retinal vein occlusion in the rat. Invest Ophthalmol Vis Sci. 2009; 50(5): 2359-2367, doi: 10.1167/iovs.08-2332, indexed in Pubmed: 18806298.

17. Ross M, Pawlina W. Eye. In: Histology: A Text and Atlas with Correlated Cell and Molecular Biology. Lippincott Williams \& Wilkins, China. 2011: 896-908.

18. Rustad OJ, Kaye V, Cerio R, et al. Postfixation of cryostat sections improves tumor definition in Mohs surgery. J Dermatol Surg Oncol. 1989; 15(12): 1262-1267, indexed in Pubmed: 2480374.

19. Schnitzer J. Chapter 7 Astrocytes in mammalian retina. Prog Retinal Res. 1988; 7: 209-231, doi: 10.1016/02784327(88)90009-0.

20. Sethi CS, Lewis GP, Fisher SK, et al. Glial remodeling and neural plasticity in human retinal detachment with proliferative vitreoretinopathy. Invest Ophthalmol Vis Sci. 2005; 46(1): 329-342, doi: 10.1167/iovs.03-0518, indexed in Pubmed: 15623793.

21. van Harreveld A, Khattab FI. Perfusion fixation with glutaraldehyde and post-fixation with osmium tetroxide for electron microscopy. J Cell Sci. 1968; 3(4): 579-594, indexed in Pubmed: 4884608. 